\title{
Optimal propofol induction dose in morbidly obese patients: bispectral index guidance is not the answer
}

\author{
Devi Chiravuri, MD • Kwaku Owusu-Bediako, MBChB • \\ Olubukola O. Nafiu, MD, FRCA, MS $\mathbb{D}$
}

Received: 14 March 2018/Accepted: 14 March 2018/Published online: 24 April 2018

(c) Canadian Anesthesiologists' Society 2018

\section{To the Editor,}

An optimal medication dosing metric for morbidly obese (MO) patients remains unresolved. Recently, Subramani et al. ${ }^{1}$ explored the possibility of bispectral index (BIS)-guided propofol dosing for induction of general anesthesia in MO patients. Two groups of patients were randomized to propofol dosing based on (1) the calculated lean body weight (LBW group) or (2) propofol infusion $100 \mathrm{mg} \cdot \mathrm{kg}^{-1} \cdot \mathrm{hr}^{-1}$ to an initial targeted BIS of 50 (BIS group). The investigators concluded that the induction dose of propofol based on BIS guidance was better for achieving predictable loss of consciousness in MO patients than the dosing based on the calculated LBW.

Unfortunately, we could not determine from their data whether the weight denominator in the BIS group was total body weight (TBW) or some other parameter. If we assume that the BIS patients received propofol based on the TBW, it is plausible that they had better predictable endpoints simply because they received more propofol than those in the LBW group. Also, previous investigators have shown that LBW dosing in MO patients provides suboptimal induction parameters. $^{2}$

Conceptually, LBW is mathematically a fraction of the TBW, so the effective drug mass based on LBW will always be lower than that based on the TBW. ${ }^{3}$ One can therefore proffer the argument that the larger effective dose of propofol (rather than BIS guidance) was responsible for

This letter is accompanied by a reply. Please see Can J Anesth 2018; 65: this issue.

D. Chiravuri, MD - K. Owusu-Bediako, MBChB .

O. O. Nafiu, MD, FRCA, MS ( $₫)$

Mott Children's Hospital, University of Michigan, Ann Arbor, MI, USA

e-mail: onafiu@med.umich.edu the smoother induction endpoint in the BIS group. This dose differential based on weight is also responsible for the large effect size of the number of patients requiring the additional doses of propofol shown in Table 3 (LBW 60\% vs BIS group 3.3\%).

Furthermore, we caution that the BIS was not designed to be an anesthetic drug "dose estimator" and suggest that the growing enthusiasm for BIS-guided dosing of propofol $^{1,2}$ be tempered with caution. The BIS was designed to monitor the brain's state of alertness and does not perform well even as a depth-of-anesthesia monitor. ${ }^{4}$ There is similar historical precedence with the widespread use of ideal body weight (IBW) dosing of medications. Just as the IBW was never intended to be a therapeutic instrument (as it is now widely used), ${ }^{5}$ currently available data simply do not support the use of BIS guidance for anesthetic drug dosing.

Although we commend the authors for their efforts at finding an optimal dosing metric for MO patients (a rapidly expanding portion of our surgical population), we suggest that caution is needed when drawing conclusions that are not supported by data from this and other studies. We suggest that requiring the inclusion of MO patients during the drug-development stage is a more inclusive, viable option that drug-licensing agencies should demand of drug developers. For now, the search continues for the optimal dosing metric in the MO patient. Unfortunately, BIS is not the answer.

Conflicts of interest None declared.

Editorial responsibility This submission was handled by Dr. Hilary P. Grocott, Editor-in-Chief, Canadian Journal of Anesthesia. 


\section{References}

1. Subramani $Y$, Riad W, Chung F, Wong J. Optimal propofol induction dose in morbidly obese patients: a randomized controlled trial comparing the bispectral index and lean body weight scalar. Can J Anesth 2017; 64: 471-9.

2. Ingrande J, Brodsky JB, Lemmens HJ. Lean body weight scalar for the anesthetic induction dose of propofol in morbidly obese subjects. Anesth Analg 2011; 113: 57-62.
3. Pai MP. Drug dosing based on weight and body surface area: mathematical assumptions and limitations in obese adults. Pharmacotherapy 2012; 32: 856-68.

4. Hajat Z, Ahmad N, Andrzejowski J. The role and limitations of EEG-based depth of anaesthesia monitoring in theatres and intensive care. Anaesthesia 2017; 72(Suppl 1): 38-47.

5. Nafiu OO, Mills K, Tremper KK. Some cautionary tales about ideal body weight dosing of anesthetic medications: it is not all that ideal! Anesth Analg 2017. DOI: https://doi.org/10.1213/ANE.0000000000002662. 